\title{
CAPCOM - Construtor de Arquiteturas Pedagógicas Colaborativas para Operações Cognitivas sobre Dados Multimídia
}

\author{
Renan F. Almeida ${ }^{1}$,Orivaldo de L. Tavares ${ }^{1}$, Luiz F. Reinoso $^{2}$ \\ ${ }^{1}$ Departamento de Informática - Universidade Federal do Espírito Santo (UFES) \\ Vitória - ES - Brasil \\ ${ }^{2}$ Coordenadoria de informática - Instituto Federal do Espírito Santo (IFES) \\ Colatina - ES - Brasil \\ ralmeida@ifes.edu.br, tavares@inf.ufes.br, luizfreinoso@gmail.com
}

\begin{abstract}
This article presents a platform to create pedagogical architectures that support collaborative dynamics to perform cognitive operations on multimedia data, collected in field activities. The platform, named CAPCOM, allows editing of educational goals (what to learn), activities (what to do) and methods (how to do), and allows the configuration of digital resources to support the development of activities. This paper presents the architecture of CAPCOM platform and two pedagogical architectures criated using that platform.
\end{abstract}

Keywords: field class;CAPCOM; pedagogical architecture; MOrFEU.

Resumo. Este artigo apresenta uma plataforma para criar arquiteturas pedagógicas que suportem dinâmicas colaborativas para executar operações cognitivas sobre dados multimídia, coletados em atividades de campo. A plataforma, denominada CAPCOM, permite a edição dos objetivos pedagógicos (o que aprender), das atividades (o que fazer) e dos métodos (como fazer), além de permitir a configuração dos recursos digitais de apoio ao desenvolvimento das atividades. Este artigo apresenta a arquitetura da plataforma CAPCOM e duas arquiteturas pedagógicas criadas via essa plataforma.

Palavras-chaves: aula de campo; CAPCOM; arquitetura pedagógica; MOrFEU.

\section{Introdução}

A aula de campo é considerada uma estratégia de ensino, tal estratégia permite articular a formação teórica e os conhecimentos práticos. Uma aula de campo permite associar as abstrações mentais aos aspectos físicos e naturais dos espaços explorados.

Aulas de campo tais como as apresentadas em [Seniciato and Cavassan 2004] e [Oliveira and Correia 2013] apresentam uma prática comumente adotada: a aplicação de avaliações após a aula. Os instrumentos usados para essas avaliações normalmente são relatos ou provas.

Quando a aula de campo for executada com apoio de recursos digitais, como aqueles disponíveis na plataforma AM (Aprendizagem em Movimento) [Pereira 2015], novas 
oportunidades de consolidar o aprendizado podem ser exploradas, com o uso de recursos digitais que permitam executar operações cognitivas sobre os dados multimídia coletados na atividade de campo.

Este artigo apresenta um espaço virtual flexível e colaborativo, intitulado CAPCOM (Construtor de Arquiteturas Pedagógicas Colaborativas para Operações Cognitivas sobre Dados Multimídia), integrado ao AM, para criação e uso de APCs (arquiteturas pedagógicas colaborativas) que permitam aos aprendizes operarem cognitivamente sobre dados multimídia registrados durante atividades de campo.

As estruturas didáticas criadas pelo professor, usando a plataforma CAPCOM, seguem o conceito de Arquitetura Pedagógica (AP). Uma Arquitetura Pedagógica é uma estrutura didático-pedagógica para potencializar a aprendizagem com o uso de recursos digitais, eventualmente em contextos de educação a distância, que se organiza em: objetivo de aprendizagem (o que aprender), atividades (o que fazer), método (como fazer) e recursos digitais (com quais ferramentas).

A modalidade de pesquisa adotada na avaliação da plataforma CAPCOM foi a aplicada, que permite comprovar ou rejeitar uma hipótese. Nessa pesquisa a hipótese defendida trata-se da seguinte: É possível colaborar com o processo de consolidação de aprendizado executando operações cognitivas sobre dados multimídia, com suporte da plataforma CAPCOM.

Este trabalho apresenta a fundamentação teórica e dois exemplos de arquiteturas pedagógicas (seção 2), define um Construtor de Arquiteturas Pedagógicas (seção 3), apresenta a plataforma CAPCOM (seção 4), trabalhos relacionados (seção 5), a avaliação da plataforma (seção 6) e as considerações finais (seção 7).

\section{Arquiteturas Pedagógicas}

Fundamentado nas Teorias de Aprendizagem, a plataforma CAPCOM adota o paradigma de AP, apoiada na pedagogia da incerteza, pressupondo que o conhecimento nasce do movimento da dúvida, da incerteza, da busca de novas alternativas, do debate e da troca. O conceito de AP estabelecendo a Pedagogia da incerteza implica em: soluções de problemas reais, transformação de informações em conhecimento, autoria, expressão e interlocução, investigação e criação de novidades e autonomia e cooperação [Carvalho et al. 2005].

Como alternativa ao ensino convencional e ao uso de apostilas para cursos a distância, uma AP abrange processos para a criação de novidades, descobertas e experimentações, centrada no ponto de vista do aprendiz, o protagonista da aprendizagem, durante a autoria de soluções individuais ou coletivas [Menezes et al. 2008].

Aprender de modo colaborativo remete a uma situação na qual duas ou mais pessoas buscam aprender algo juntas. Essa proposta é considerada muito relevante para apoiar o processo de aprendizado [Dillenbourg et al. 1995].

Desenvolver atividades colaborativas é a "condição necessária para que as redes de aprendizagem se constituam e se mantenham, em prol de concretizar a construção de conhecimento pelos seus intervenientes" [Chagas 2002].

O modelo 3C de colaboração - comunicação, coordenação e cooperação 
V Congresso Brasileiro de Informática na Educação (CBIE 2016)

Anais dos Workshops do V Congresso Brasileiro de Informática na Educação (CBIE 2016)

[Pimentel et al. 2006], foi utilizado como referência para descrever os recursos digitais que dão suporte a colaboração na plataforma CAPCOM. A seguir são apresentadas duas arquiteturas pedagógicas que podem ser criadas na plataforma.

- Nome da AP: Arquitetura Pedagógica Associando Conceito.

- Contexto: A partir de dados multimídia coletados por um grupo de alunos em visita a um museu, um professor define um conjunto de conceitos relacionados aos conhecimentos articulados durante a visita e solicita que os alunos associem as mídias produzidas à lista de conceitos criados pelo professor.

- Objetivo de aprendizagem: Aprender a classificar dados multimídia, de acordo com os conceitos propostos pelo professor.

- Atividades: Associar dados multimídia produzidos em uma aula de campo (neste caso, uma visita a um museu) a um ou vários conceitos e visualizar as associações no formato de mapas mentais.

- Método: O professor cria um conceito que precisa ser explorado pelos alunos, forma grupos de três alunos e solicita que os alunos colaborem para associar todas as mídias produzidas na aula de campo, que tenham relação com aquele conceito.

- Recurso digital: São usados recursos para visualizar mapas mentais, classificação de dados multimídia, suporte a grupos de colaboração, chat, grupo e edição de dados multimídia.

- Colaboração: No recurso digital "Mapa Mental" os alunos trabalham com a fase de cooperação para criar as associações visualizadas em mapa mental. No recurso "Grupo" os alunos e o professor trabalham a fase de coordenação e comunicação para viabilizar os resultados a serem produzidos. No recurso "Classificar" os alunos trabalham a fase de cooperação para compor os novos dados multimídia. Nos recursos chat e suporte à colaboração os alunos trabalham a fase de coordenação e comunicação.

- Nome da AP: Arquitetura Pedagógica Guia Digital.

- Contexto: Após uma aula de campo, que acontece em uma reserva natural, o professor decide criar atividades pedagógicas colaborativas para os alunos relatarem os conhecimentos adquiridos, usando as mídias produzidas e registradas durante a aula.

- Objetivo de aprendizagem: Aprender a descrever a experiência de aprendizado vivenciada durante uma aula de campo.

- Atividades: Combinar logicamente os dados multimídia e produzir uma apresentação das atividades desenvolvidas durante a aula de campo.

- Método: O professor cria um grupo de três alunos e desafia o grupo a produzir uma apresentação das atividades conduzidas durante a aula de campo.

- Recurso digital: São utilizados recursos para edição de dados multimídia, suporte a colaboração, chat, grupo e negociação para uso de dados multimídia.

- Colaboração: Com o recurso "Relato" os alunos trabalham a fase de cooperação para compor novos dados multimídia. Com o recurso "Grupo" os alunos e o professor trabalham a fase de coordenação e comunicação para viabilizar os resultados a serem produzidos. Com o recurso digital "Negociação de Mídia", os alunos e o professor trabalham a fase da comunicação, coordenação e cooperação em conjunto, para viabilizar o uso de uma mídia, de autoria de um aluno, por parte de outro aluno do mesmo grupo. Nos recursos chat e suporte à colaboração os alunos trabalham a fase de coordenação e comunicação. 
V Congresso Brasileiro de Informática na Educação (CBIE 2016)

Anais dos Workshops do V Congresso Brasileiro de Informática na Educação (CBIE 2016)

\section{Construtor de Arquiteturas Pedagógicas}

Um Construtor de Arquitetura Pedagógicas (CAP) suporta a criação de arquiteturas pedagógicas, auxiliando o educador, mesmo que leigo em informática, a criar as atividades e implementar os recursos digitais de uma ou mais APs definidas por ele [Reinoso 2016].

A CAPCOM é uma especialização do CAP, pois permite criar APs para executar operações cognitivas sobre dados multimídia, coletados em atividades de campo. Esse contexto de aplicação representa uma dentre várias possibilidades. Usando o CAP é possível criar APs para qualquer contexto de aprendizagem.

A plataforma CAPCOM permite a construção de APs, de tal modo que o professor pode instrumentalizar suas APs com recursos digitais especialmente configurados por ele, sem que o professor precise saber programação. Para permitir essa flexibilidade, a CAPCOM implementa conceitos do projeto MOrFEU, tais como: plasticidade, ergonomia, redução da repetição de trabalho e redução da sobrecarga cognitiva [Menezes et al. 2008].

Ao invés de buscar ferramentas que possam atender as necessidades de uma AP, o professor configura esses recursos diretamente na plataforma CAPCOM.

Os conceitos associados ao MOrFEU, implementados na plataforma CAPCOM, permitem ao professor a flexibilidade de criar os recursos digitais usados nas atividades. Essas atividades buscam desafiar os aprendizes a realizarem operações cognitivas sobre dados multimídia, tais como: fotos, textos, áudio etc. Na implementação da CAPCOM, são usados três conceitos do MOrFEU: VCom (Veículo de Comunicação); UPI (Unidade de Produção Intelectual) e Template para VCom.

Um VCom é um veículo de publicação de textos (multimídia). Em um VCom as pessoas assumem papéis, as vezes simultaneamente, de criadores e consumidores de conteúdo [Pereira 2015]. Alguns exemplos que materializam o conceito de VComs são: redes sociais digitais, revista, relatório, mapas mentais etc.

"O conceito de UPI (unidade de produção intelectual) permite que todo texto (uma foto, um texto escrito, um vídeo, um áudio etc) seja registrado com autoria, data, versão etc, de modo independente de onde ela esteja publicada" [Vieira et al. 2011]. Uma UPI é a unidade base de toda informação produzida e processada na plataforma CAPCOM.

O conceito de Template para VCom permite configurar diferentes formas visuais para um mesmo conjunto de UPIs associados a um VCom. "Cada VCom pode estar associado a diferentes templates, o que permite que cada usuário possa definir como deseja visualizá-lo" [Vieira 2011]. Como exemplo, um relato de campo, do tipo VCom, pode assumir vários arranjos simultâneos, podendo ser constituído de imagens, textos etc.

\section{A plataforma CAPCOM}

Nesta seção são apresentadas as características da CAPCOM, implementada com o objetivo de auxiliar os alunos no processo de consolidação dos conhecimentos adquiridos em uma aula de campo.

Usando a CAPCOM o professor pode configurar os recursos digitais de suas APs. Tais recursos permitem processar diferentes tipos de dados multimídia (UPIs). As configurações aplicadas sobre os recursos permitem modificar seu comportamento, proporcionando flexibilidade para ajustá-lo às necessidades de cada AP. 
V Congresso Brasileiro de Informática na Educação (CBIE 2016)

Anais dos Workshops do V Congresso Brasileiro de Informática na Educação (CBIE 2016)

Os usuários típicos da plataforma são: o professor, o aluno e o visitante. O professor administra a CAPCOM para criar suas APs, configurar os recursos digitais e visitar as UPIs produzidas pelos alunos.

O aluno, usando os "Recursos Digitais", desenvolve colaborativamente as atividades propostas em cada AP, criando ou combinando UPIs. Os visitantes utilizam o "Mural" para visualizar as UPIs produzidas pelos alunos.

A plataforma CAPCOM suporta a construção de diversas APs, além das duas apresentadas na seção 3. Os recursos digitais disponíveis na plataforma podem ser usados e configurados para atender as necessidades de cada AP. No texto a seguir são apresentados os recursos disponíveis:

1. Classificar: permite associar UPIs com um conceito.

2. Relato: esse recurso permite a construção de um relato (um VCom), a partir da combinação de UPIs que podem ser de diferentes mídias.

3. Mapa Mental: permite visualizar, em forma de mapa mental, as associações criadas com o recurso "Classificar".

4. Grupo: permite criar e gerenciar grupos de colaboração.

5. Negociação de Mídia: usando esse recurso, um usuário pode solicitar o uso de uma mídia (UPI) que não seja de sua autoria.

6. Incluir Mídia: com esse recurso os usuários podem incluir novas mídias (UPIs).

7. Chat: permite aos alunos se comunicarem via texto.

8. Mensagem: permite aos alunos trocarem mensagens entre si.

9. Tarefas: permite aos alunos criarem listas de tarefas.

Além dos recursos listados nesta seção, a plataforma CAPCOM também permite restringir as UPIs processadas, ou seja, o professor pode definir que um determinado recurso só pode ser utilizado para processar um conjunto especifico de UPIs. Os tipos de restrições suportadas pela plataforma são: por UPI, tipo de UPI, autor, localidade e tempo.

Para criar uma AP na plataforma CAPCOM, o professor descreve o objetivo de aprendizagem, a atividade pedagógica e a metodologia, em texto escrito. Após preencher essas informações, o professor seleciona recursos disponíveis na plataforma e configura o comportamento do Recurso Digital criado, de acordo com a AP definida por ele.

A Figura 1 apresenta um exemplo de atividade produzida com a biblioteca "Relato", onde é possível visualizar uma das páginas que compõe uma atividade feita por um usuário da AP "Guia Digital”. Na Figura 2 é apresentado o resultado obtido com o uso da biblioteca "Classificar", usando o formato visual do recurso digital "Mapa Mental", na AP "Associando Conceitos". 
V Congresso Brasileiro de Informática na Educação (CBIE 2016)

Anais dos Workshops do V Congresso Brasileiro de Informática na Educação (CBIE 2016)

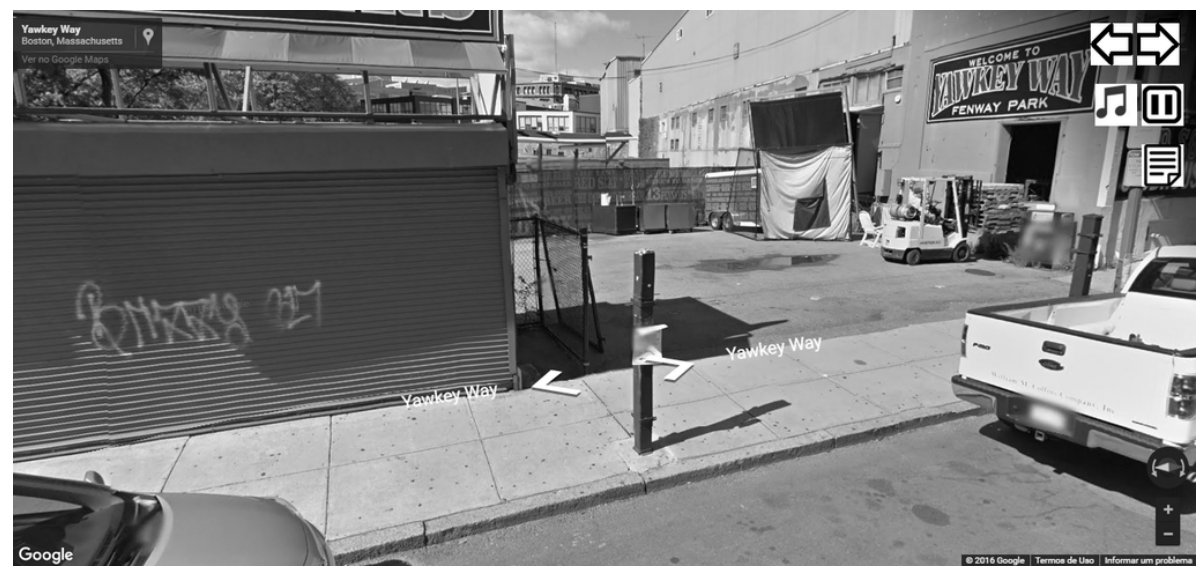

Figura 1. Página de relato gerada em uma AP.

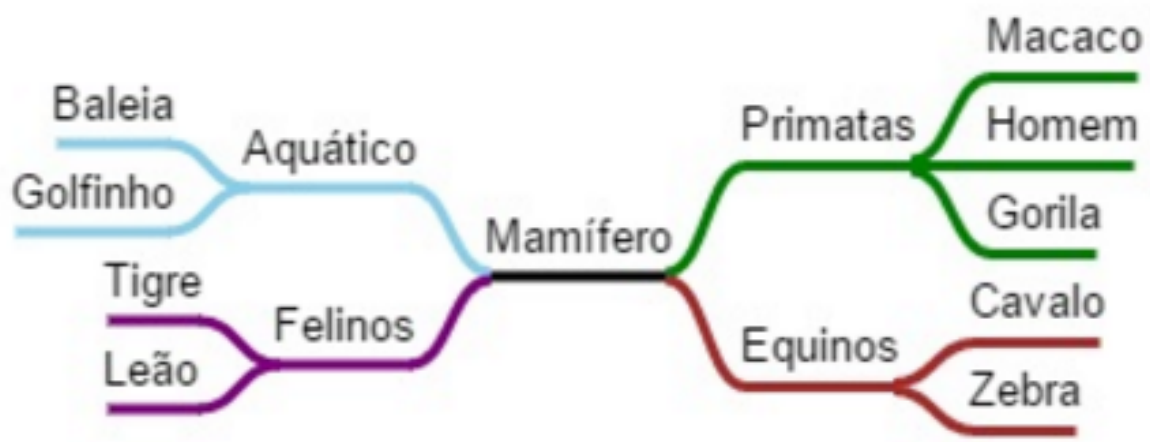

Figura 2. Mapa mental gerado em uma AP.

\section{Trabalhos Relacionados}

A Tabela 1 apresenta uma comparação da plataforma CAPCOM com outros sistemas. Na coluna "Projetado para" é apresentado o(s) modo(s) de uso do sistema: "Móvel" significa que o sistema foi desenvolvido para usar recursos de dispositivos móveis e "Web" indica que o sistema foi construído para usar recursos da Web.

A coluna "Principal Uso" apresenta o propósito principal de construção do sistema. A coluna "Colaboração" define se o sistema suporta a colaboração durante a utilização, dentre as diversas possibilidades oferecidas. A coluna "Configurável" indica se o sistema permite configurar, de forma flexível, atividades pedagógicas.

Tabela 1. Comparação entre sistemas digitais para atividades pedagógicas.

$\begin{array}{lllll}\text { Sistema } & \text { Projetado para } & \text { Principal uso } & \text { Colaboração } & \text { Configurável } \\ \text { Aris Games } & \text { Móvel } & \text { Jogos } & \text { Não } & \text { Sim } \\ \text { CriEduc } & \text { Web } & \text { Educacional } & \text { Sim } & \text { Sim } \\ \text { Youubi } & \text { Móvel } & \text { Educacional } & \text { Sim } & \text { Não } \\ \text { CAPCOM } & \text { Web } & \text { Educacional } & \text { Sim } & \text { Sim }\end{array}$


V Congresso Brasileiro de Informática na Educação (CBIE 2016)

Anais dos Workshops do V Congresso Brasileiro de Informática na Educação (CBIE 2016)

A plataforma Youubi [Monteiro et al. 2015] é um ambiente de aprendizado ubíquo, criado para suportar práticas pedagógicas em espaços abertos, usando dispositivos móveis. A plataforma Youubi e a plataforma CAPCOM permitem criar atividades para consolidação de conhecimento, porém a CAPCOM permite criar várias atividades, em APs, usando os recursos digitais disponíveis, enquanto a Youиbi permite criar apenas atividades com questões de múltipla escolha.

A plataforma CriEduc [Bergami and Tavares 2015] foi projetada para suportar passeios virtuais com apoio de tecnologias georreferenciadas, semelhante as aulas de campo, tal plataforma e a CAPCOM guardam semelhanças, ambas permitem criar arquiteturas pedagógicas colaborativas. A modelagem adotada na implementação, para flexibilizar a configuração das APs, é diferente nas duas plataformas, a CAPCOM implementa o conceito CAP os conceitos que fundamentam o MOrFEU. A plataforma CriEduc se propõem a atender a um público diferente da plataforma CAPCOM, sendo que a CAP$\mathrm{COM}$ foi projetada, a princípio, para suportar práticas pedagógicas que acontecem após uma aula de campo.

Como a CAPCOM adota a modelagem do MOrFEU [Menezes et al. 2008], ela permite criar e ajustar as arquiteturas pedagógicas e os recursos digitais a cada contexto.

\section{Avaliação da Plataforma}

Por meio de uma pesquisa aplicada, foi levantada a seguinte hipótese para avaliar a plataforma: "É possível colaborar com o processo de consolidação do aprendizado de estudantes, com o uso de dados multimídia coletados em uma aula de campo, ao executarem operações cognitivas sobre esses dados, com o suporte da plataforma CAPCOM".

Para validar a proposta foi feita uma pesquisa quantitativa com sete pessoas, dois professores e cinco alunos, que foram convidadas a usar a plataforma para instrumentalizar suas APs. Os participantes com perfil de professor são educadores e trabalham no Instituto Federal do Espírito Santo (IFES) Campus Colatina, os participantes com perfil de aluno cursam Bacharelado em Sistemas de Informação, também no IFES Campus Colatina. A atividade de referência, para a avaliação, foi uma visita a um museu, nessa visita foram produzidos vários dados multimídia.

Após os alunos participarem das atividades pedagógicas, instrumentalizadas pelos professores, eles preencheram um formulário no qual indicavam quais informações foram abordadas durante as atividades pedagógicas, o formulário tinha 29 assertivas, 15 verdadeiras e 14 falsas, de $100 \%$ das assertivas selecionadas pelos alunos, $76 \%$ estavam corretas.

Como a avaliação aconteceu com um grupo reduzido, os números traduzem um indicativo de que a hipótese levantada pode ser verdadeira, e que o uso da plataforma CAPCOM permite a criação e uso de APs que podem favorecer a consolidação de conhecimento, a partir do uso de dados multimídia. Em particular, essas APs podem ter o objetivo de favorecer a consolidação de conhecimento sobre atividades realizadas em campo, onde tenha ocorrido a coleta de dados multimídia.

\section{Considerações Finais}

A plataforma CAPCOM foi projetada para completar o ciclo pedagógico de uma aula de campo que é: planejamento da aula de campo, a aula e as atividades de consolidação 
V Congresso Brasileiro de Informática na Educação (CBIE 2016)

Anais dos Workshops do V Congresso Brasileiro de Informática na Educação (CBIE 2016)

do conhecimento posteriores à aula de campo. Para completar esse ciclo, este artigo apresentou uma plataforma que tem o potencial de auxiliar os alunos a consolidarem os conhecimentos adquiridos durante uma atividade de campo, permitindo que eles executem operações cognitivas sobre os dados multimídia produzidos durante a aula, de modo colaborativo.

Apesar do propósito inicial da concepção da plataforma CAPCOM, constata-se que os dados multimídias operados pelos recursos digitais da CAPCOM podem ter sido coletados de diversos modos, não apenas durante uma aula de campo. Assim, as atividades propostas na APs podem envolver os estudantes em operações cognitivas sobre quaisquer dados multimídia.

Foram apresentadas, como exemplo, duas APs que podem ser criadas pelo professor, por meio da CAPCOM. As APs apresentadas indicam que a plataforma e seus recursos digitais podem atender a um conjunto de atividades pedagógicas, baseadas em operações cognitivas sobre dados multimídia.

Como trabalhos futuros, pretende-se expandir o suporte a novos tipos de UPIs, incluindo vídeos esféricos e outras mídias ainda pouco exploradas, mas que tenham o potencial de auxiliar os aprendizes no processo de consolidação dos conhecimentos adquiridos em aulas de campo.

\section{Referências}

Bergami, W. V. and Tavares, O. L. (2015). Crieduc - sistema web de aprendizagem com tecnologias geoespaciais. In XX Congreso Internacional de Informática Educativa, volume 11, pages $315-322$.

Carvalho, M. J. S., Nevado, R. A. d., and Menezes, C. d. (2005). Arquiteturas pedagógicas para educação a distância: concepções e suporte telemático. Anais do XVI Simpósio Brasileiro de Informática na Educação, Juiz de Fora-MG. Brasil, pages 351-360.

Chagas, I. (2002). Trabalho colaborativo condição necessária para a sustentabilidade das redes de aprendizagem. Redes de aprendizagem. Redes de conhecimento. Lisboa: Conselho Nacional de Educação, pages 71-82.

Dillenbourg, P., Baker, M. J., Blaye, A., and O’Malley, C. (1995). The evolution of research on collaborative learning. Learning in Humans and Machine: Towards an interdisciplinary learning science., pages 189-211.

Menezes, C. S., de Nevado, R. A., de Castro Jr, A. N., and Santos, L. N. (2008). Morfeumulti-organizador flexível de espaços virtuais para apoiar a inovação pedagógica em ead. In Anais do Simpósio Brasileiro de Informática na Educação, volume 1, pages 451-460.

Monteiro, B. d. S., de Oliveira, E. J., Gomes, A. S., and Mendes, F. N. (2015). Youubi: Ambiente de aprendizagem ubíqua. In Anais do Simpósio Brasileiro de Informática na Educação, volume 26, page 111.

Oliveira, A. and Correia, M. D. (2013). Aula de campo como mecanismo facilitador do ensino-aprendizagem sobre os ecossistemas recifais em alagoas. Alexandria Revista de Educação em Ciência e Tecnologia, 6(2):163-190. 
V Congresso Brasileiro de Informática na Educação (CBIE 2016)

Anais dos Workshops do V Congresso Brasileiro de Informática na Educação (CBIE 2016)

Pereira, B. C. (2015). Aprendizagem em movimento - uma plataforma para a criaçção e uso de veiculos de comunicação na realização de arquiteturas pedagógicas. Master's thesis, Programa de pós graduação em em Informática da UFES, Vitória-ES.

Pimentel, M., Gerosa, M. A., Filippo, D., Raposo, A., Fuks, H., and Lucena, C. J. P. (2006). Modelo 3c de colaboração para o desenvolvimento de sistemas colaborativos. Anais do III Simpósio Brasileiro de Sistemas Colaborativos, pages 58-67.

Reinoso, L. F. (2016). Uma plataforma para construção e uso de arquiteturas pedagógicas para aprendizagem de libras. Master's thesis, Dissertação (Mestrado em Informática)UFES, Vitória-ES, Maio.

Seniciato, T. and Cavassan, O. (2004). Aulas de campo em ambientes naturais e aprendizagem em ciências-um estudo com alunos do ensino fundamental field classes in natural environment and science learning-a study with students from elementary school. Ciência \& Educação, 10(1):133-147.

Vieira, R. R. M. (2011). Uma arquitetura de software para o morfeu: Apoiando a realização de arquiteturas pedagógicas em espaços virtuais colaborativos. Master's thesis, Dissertação (Mestrado em Informática)-UFES, Vitória-ES, Agosto.

Vieira, R. R. M., dos Santos, O. L., do Prado Rafalski, J., Bada, E. M., da Silva, H. F. A., and de Menezes, C. S. (2011). Coordenação nas atividades colaborativas em ambientes de aprendizagem-uma avaliação na implementação de arquiteturas pedagógicas. RENOTE, 9(1). 